\title{
A Total Synthesis of $( \pm)$-3-O-Demethylmacronine Through Rearrangement of a Precursor Embodying the Haemanthidine Alkaloid Framework
}

\author{
Xiang Ma, Nadia Gao, Martin G. Banwell,* Paul D. Carr and \\ Anthony C. Willis
}

Research School of Chemistry, Institute of Advanced Studies

The Australian National University, Canberra, ACT 2601, Australia

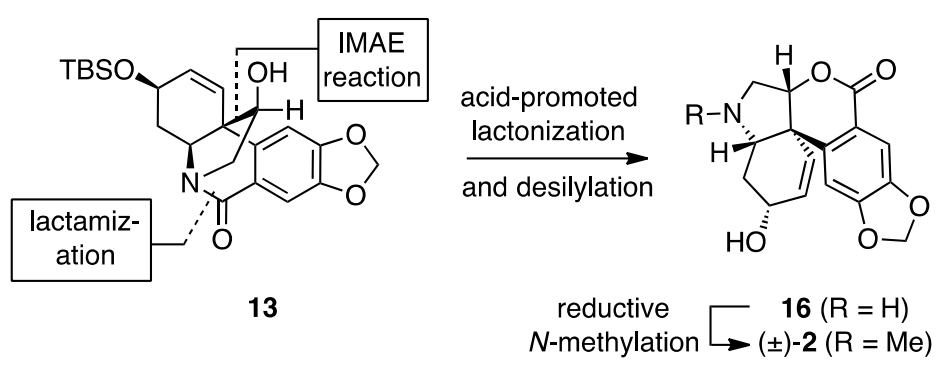

\begin{abstract}
A total synthesis of the racemic modification, $( \pm)-2$, of the tazettine-type alkaloid 3-O-demethylmacronine is described. The key steps are an intramolecular Alder-ene (IMAE) reaction and a lactam-to-lactone rearrangement of tetracycle 13, a compound that embodies the haemanthidine alkaloid framework.
\end{abstract}




\section{INTRODUCTION}

In 1964 Hauth and Stauffacher reported ${ }^{1}$ the isolation of the alkaloid macronine (1) (Figure 1) from the plant Crinum macrantherum Engl. (Amaryllidaceae) and the assignment of its full structure, by Wildman and co-workers, ${ }^{2}$ followed shortly thereafter. The latter group noted that compound $\mathbf{1}$ represents the first example of a lactonic Amaryllidaceae alkaloid possessing the tazettine ring system. They also revealed that a strained lactam incorporated within the haemanthidine alkaloid framework rearranged to give $N$-demethylmacronine in buffer at $\mathrm{pH} 6.80 .^{2 \mathrm{a}}$ Whether or not such a rearrangement has biosynthetic relevance remains unclear. In 1999 Hesse and co-workers described ${ }^{3}$ the isolation of 3-O-demethylmacronine (2) from a Galanthus species of Turkish origin and the illustrated structure was established using conventional NMR spectroscopic methods. The same group also determined that the compound does not arise through demethylation of congener $\mathbf{1}$ during the isolation process. Accordingly, 3-O-demethylmacronine (2) is considered to be a naturally occurring alkaloid.
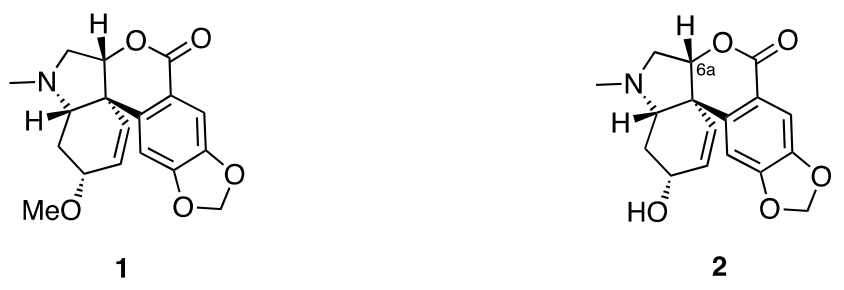

Figure 1: The structures the alkaloid macronine (1) and its 3-O-demethyl congener (2).

Thus far, no biological evaluation of compound $\mathbf{2}$ has been reported. Furthermore, while macronine (1) has been isolated from a range of plant sources since $1964,{ }^{4}$ studies of its potential therapeutic properties appear to have been confined to ones utilizing crude extracts 
of the producing plants and thus suggesting that it may possess, at a minimum, useful antibacterial and/or anti-fungal properties. ${ }^{4 \mathrm{~d}, 4 \mathrm{e}}$

In 1976 Tsuda et al reported ${ }^{5}$ a ca. 14-step synthesis of $( \pm)$-macronine that exploited, as a late-stage transformation, a rearrangement reaction of the type described by Wildman. No other relevant work on alkaloids $\mathbf{1}$ or $\mathbf{2}$ has been reported since then and nor do there appear to have been any studies on the stereochemical requirements (if any) of this pivotal and potentially versatile rearrangement process. It is against this background that we now report a ten-step synthesis of $( \pm)$-3-O-demethylmacronine [ $\pm-(2)]$ from readily available materials and also detail a synthesis of its C6a-epimer through an analogous but even more facile rearrangement reaction.

\section{RESULTS AND DISCUSSION}

The synthetic route used to obtain the requisite, strained lactam embedded within the haemanthidine alkaloid framework is shown in Scheme 1. Thus, Suzuki-Miyaura crosscoupling of the known boronate ester $3^{6}$ with the previously reported cycloalkenyl bromide $\mathbf{4}^{7}$ gave the arylated cyclohexene 5 (83\%) and this was readily propargylated at nitrogen using 1-bromo-2-butyne in the presence of sodium hydride to give derivative 6 in $91 \%$ yield. This last compound participated in a intramolecular Alder-ene (IMAE) reaction, ${ }^{8}$ using $\operatorname{Pd}(\mathrm{OAc})_{2}$ and the strong $\sigma$-donating ligand $N, N^{\prime}$-bis(benzylidene)ethylenediamine (BBEDA) in refluxing toluene, thereby affording the C3a-arylated hexahydroindole 7 in $73 \%$ yield. This IMAE product was accompanied by small amounts of uncharacterized materials, one of 
which is likely to the isomeric cyclopropyl-containing system analogous to that observed ${ }^{6}$ when the tosyl analogue of substrate $\mathbf{6}$ was subjected to the same type of reaction.

Scheme 1: Synthesis of the strained keto-lactam 9 embodying the haemanthidine alkaloid framework

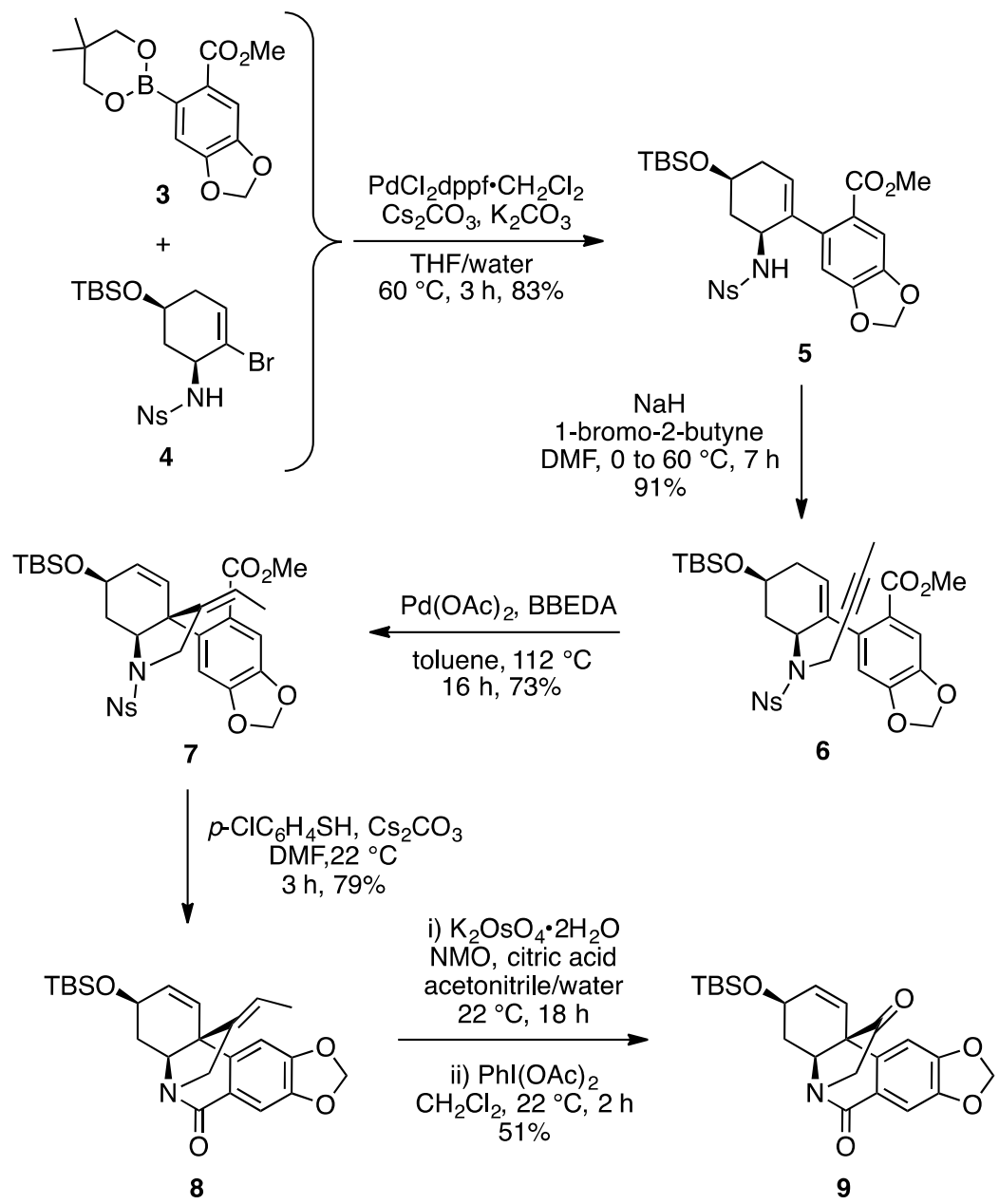

Subjection of compound $\mathbf{7}$ to reaction with $p$-chlorobenzenethiol and cesium carbonate in dimethylformamide (DMF) at ambient temperatures, conditions defined by Fukuyama for the cleavage of nosylates, ${ }^{9}$ not only resulted in removal of the sulfonamide residue but also 
effected a lactamization reaction involving the pendant ester residue thus leading to compound 8 (79\%), the structure of which was secured by single-crystal X-ray analysis [see Experimental Section and the Supporting Information (SI) for details]. The exocyclic double bond associated with lactam $\mathbf{8}$ could be oxidatively cleaved through its initial and selective dihydroxylation under conditions defined by Bäckvall ${ }^{10}$ and then subjecting the cis-vicinal diol so-formed to treatment with iodosobenzene diacetate. ${ }^{11}$ By such means the ketone $\mathbf{9}$ was obtained in 51\% yield over the two steps involved. Compound 9 displays a lactam carbonyl absorption band at $1700 \mathrm{~cm}^{-1}$ in the infra-red spectrum while in the ${ }^{13} \mathrm{C}$ NMR spectrum of this same material the associated carbon resonates at $\delta 179.8 \mathrm{ppm}$. These values stand as testimony to the strained nature of this nitrogen-containing ring system (the equivalent values for $\delta$-valerolactam are ca. $1672 \mathrm{~cm}-1$ and $\delta \square 169.1$, respectively ${ }^{12}$ ).

When a methanolic solution of keto-lactam 9 maintained at $0{ }^{\circ} \mathrm{C}$ was treated with sodium borohydride non-stereoselective reduction of the associated ketone residue took place to afford a chromatographically separable mixture of compounds $\mathbf{1 0}$ and $\mathbf{1 1}$ (Scheme 2). Since each of these reduction products was obtained as an interconverting mixture of epimers/anomers they were subjected, as a mixture and without extensive spectroscopic characterization, to oxidation with manganese dioxide and thereby affording the chromatographically separable lactone 12 (45\% from 9) and lactam 13 (29\% from 9), respectively. The structure of compound $\mathbf{1 3}$ was confirmed by single-crystal X-ray analysis (see Experimental Section and SI for details). Presumably compound $\mathbf{1 0}$ arises through initial reduction of the ketone carbonyl residue within precursor $\mathbf{9}$ such that the hydroxyl group within the resulting alcohol sits, as is evident from inspection of molecular models, directly 
above lactam carbonyl moiety and can thus approach the latter along a Bürgi-Dunitz trajectory ${ }^{13}$ and so facilitating conversion into the isomeric lactone that is itself reduced to the observed mixture of lactols $\mathbf{1 0}$. In contrast, the epimeric alcohol arising from reduction of the ketone residue within compound $\mathbf{9}$ cannot so readily engage in a lactam-to-lactone isomerization process and thus the residual (and strained) lactam carbonyl group is reduced directly to give compound $\mathbf{1 1}$.

Scheme 2: Chemical manipulation of compound 9 leading to lactone 12 and lactam 13

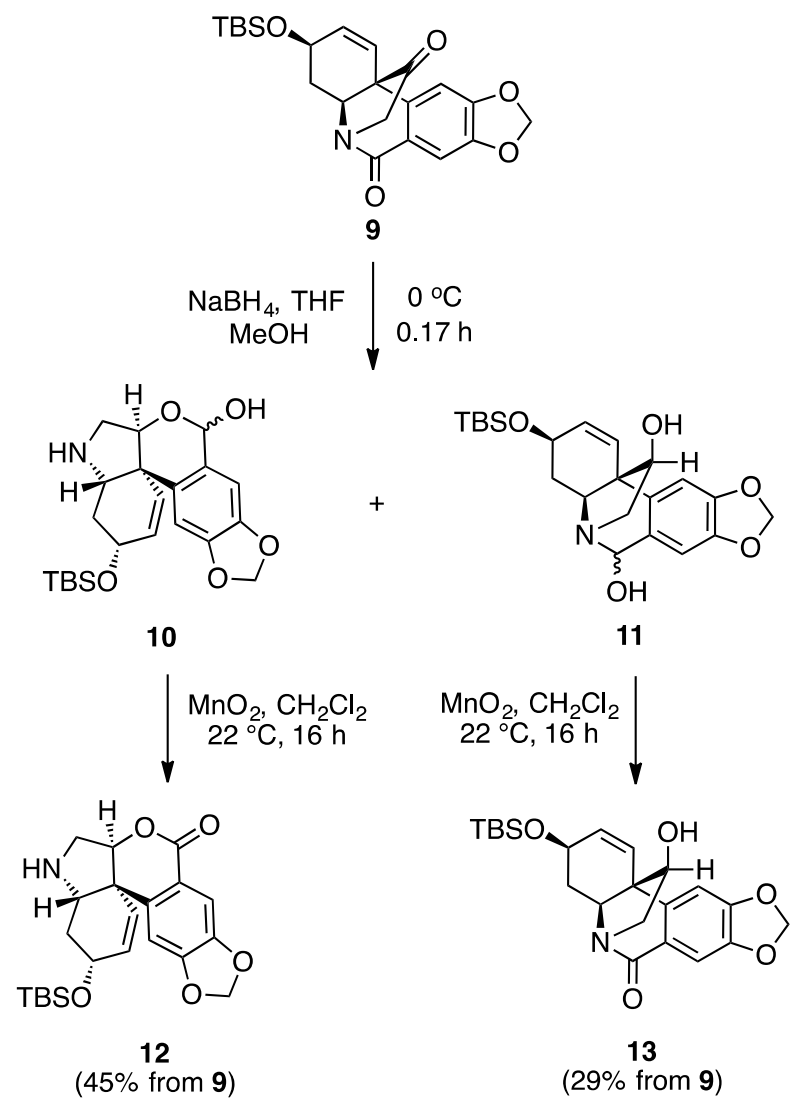

The simple synthetic pathway used to convert lactone 12 into compound 15, the C6a-epimer of ( \pm )-3-O-demethylmacronine, is shown in Scheme 3. Thus, treatment of silyl ether $\mathbf{1 2}$ with 
HF•pyridine in THF at ambient temperatures for one hour gave the expected allylic alcohol 14 in 99\% yield and when this was treated with potassium hydride and methyl iodide in THF at $0{ }^{\circ} \mathrm{C}$ for $0.5 \mathrm{~h}$ then the anticipated $3^{\circ}$-amine $\mathbf{1 5}$ was obtained in near quantitative yield. Interestingly, in the second step of this reaction sequence no product arising from $O$ methylation of the allyic alcohol moiety was observed. The ${ }^{1} \mathrm{H}$ and ${ }^{13} \mathrm{C}$ NMR spectral data acquired on compound $\mathbf{1 5}$ were in complete accord with the assigned structure and quite distinct from those recorded for the natural product $2 .^{3}$

Scheme 3: Conversion of lactone 12 into the C6a-epimer, 15, of ( \pm )-3-O-demethylmacronine $[( \pm)-2]$
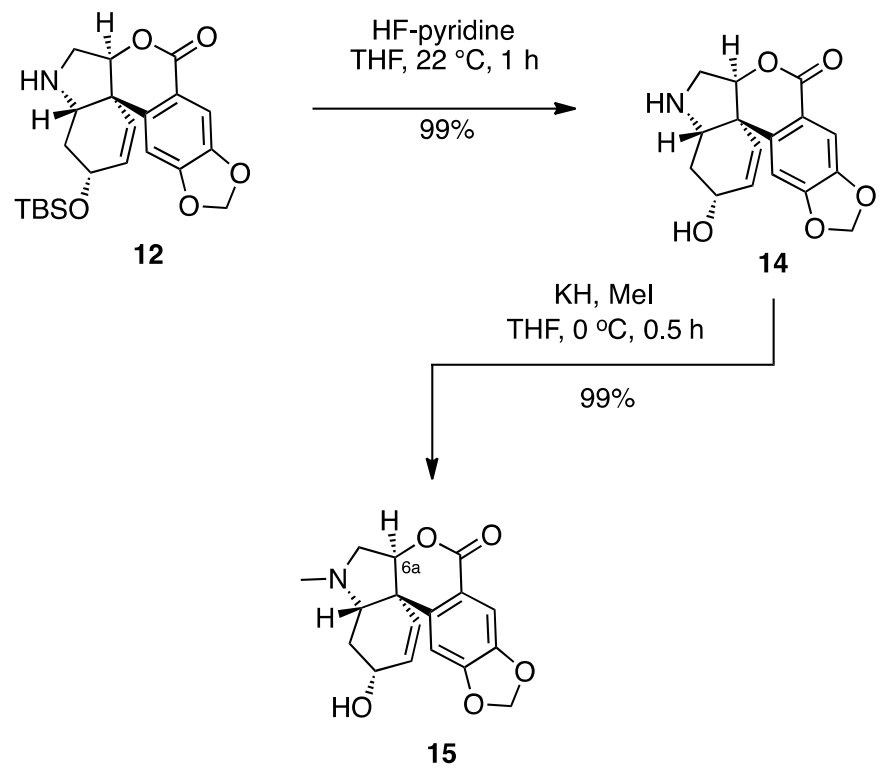

The route used in completing the total synthesis of target ( \pm )-2 is shown in Scheme 4 . This involved, as a pivotal step, the p-toluenesulfonic acid acid-promoted conversion of the haemanthidine-based hydroxylactam 13 into the lactone 16 (48\%) and as part of this process 
the silyl ether associated with the starting material was cleaved. The precise pathway by which this rearrangement takes place remains unclear. However, given the likely abnormally basic nature of the nitrogen associated with the bridged lactam ${ }^{14}$ in substrate $\mathbf{1 3}$ protonation at this center followed by cleavage of the $\mathrm{N}-\mathrm{C}=\mathrm{O}$ single bond followed by reaction of the resulting acylium ion with the pendant hydroxyl group would afford the observed lactone $\mathbf{1 6 .}$ Reductive $N$-methylation of compound $\mathbf{1 6}$ using sodium cyanoborohydride and formaldehyde in acetic acid at ambient temperatures then gave $( \pm)$-3- $O$-demethylmacronine $[( \pm)-2]$ in $89 \%$ yield. Interestingly, attempts to effect the $O$-methylation of the last compound under a range of conditions ${ }^{15}$ failed to generate $( \pm)$-macronine $[( \pm)-1]$. While the origins of this situation are not clear the likely close spatial arrangement of the hydroxy and amine groups within compound $( \pm)-2$ could be responsible.

Scheme 4: Completion of the total synthesis of $( \pm)$-3-O-demethylmacronine $[( \pm)-2]$

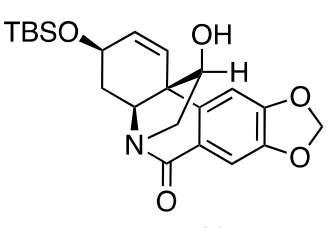

13

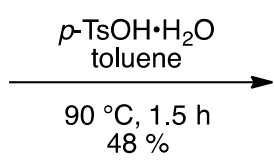

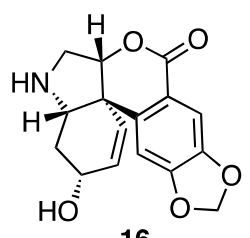

16
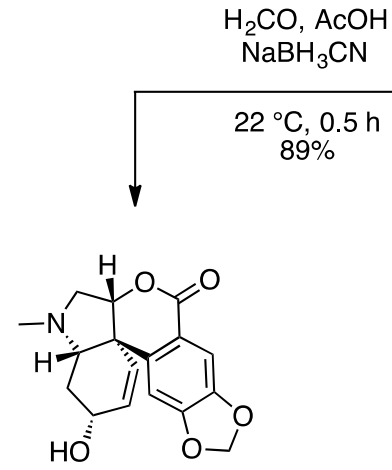

(士)-2 
All the spectral data acquired on compound $( \pm)$-2 were in complete accord with the assigned structure while the ${ }^{1} \mathrm{H}$ and ${ }^{13} \mathrm{C}$ NMR spectra recorded on the synthetic material matched those reported by Hesse ${ }^{3}$ for the natural product (see SI for tabulated comparisons).

\section{CONCLUSION}

The results reported here demonstrate that strained lactam units embedded within a C12hydroxylated haemanthidine framework can be engaged in rearrangement reactions that generate the tetracyclic skeleton of the macronine alkaloids. These rearrangements appear to proceed regardless of the stereochemistry at C-12, although the reaction pathways involved are quite different in each instance. While the biosynthetic relevance (or otherwise) of such

processes remain to be determined, they are likely to enable the preparation of a range of frameworks of biological interest. Work directed towards examining such possibilities is now underway. 


\section{EXPERIMENTAL SECTION}

General Protocols. Unless otherwise specified, proton $\left({ }^{1} \mathrm{H}\right)$ and carbon $\left({ }^{13} \mathrm{C}\right)$ NMR spectra were recorded at $18{ }^{\circ} \mathrm{C}$ in base-filtered $\mathrm{CDCl}_{3}$ on a spectrometer operating at $400 \mathrm{MHz}$ for proton and $100 \mathrm{MHz}$ for carbon nuclei. ${ }^{1} \mathrm{H}$ NMR data are recorded as follows: chemical shift

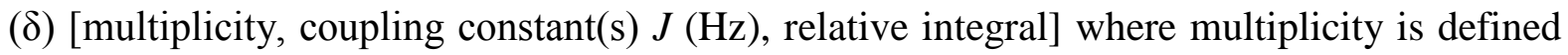
as $\mathrm{s}=$ singlet; $\mathrm{d}=$ doublet; $\mathrm{t}=$ triplet; $\mathrm{q}=$ quartet; $\mathrm{m}=$ multiplet or combinations of the above. In relevant cases, the signal due to residual $\mathrm{CHCl}_{3}$ appearing at $\delta_{\mathrm{H}} 7.26$ and the central resonance of the $\mathrm{CDCl}_{3}$ "triplet" appearing at $\delta_{\mathrm{C}} 77.0$ were used to reference ${ }^{1} \mathrm{H}$ and ${ }^{13} \mathrm{C}$ NMR spectra, respectively. Samples were analyzed by infrared spectroscopy $\left(v_{\max }\right)$ as thin films on $\mathrm{KBr}$ plates or as neat material resting on the sampling port. Low- and highresolution electron impact (EI) mass spectra were recorded on a double-focusing, triplesector machine. Low- and high-resolution ESI mass spectra were recorded on a triplequadrupole mass spectrometer operating in either positive or negative ion mode. Melting points are uncorrected. Analytical thin layer chromatography (TLC) was performed on aluminum-backed $0.2 \mathrm{~mm}$ thick silica gel $60 \mathrm{~F}_{254}$ plates. Eluted plates were visualized using a $254 \mathrm{~nm}$ UV lamp and/or by treatment with a suitable dip followed by heating. These dips included phosphomolybdic acid/ceric sulfate/sulfuric acid (conc.)/water (37.5 g : 7.5 g : 37.5 g : $720 \mathrm{~mL}$ ), potassium permanganate/potassium carbonate/5\% sodium hydroxide aqueous solution/water (3 g : $20 \mathrm{~g}: 5 \mathrm{~mL}: 300 \mathrm{~mL}$ ), and p-anisaldehyde or vanillin/sulfuric acid (conc.)/ethanol (15 g : $2.5 \mathrm{~mL}$ : $250 \mathrm{~mL}$ ). Flash chromatographic separations were carried out following protocols defined by Still et al. ${ }^{16}$ with silica gel $60(40-63 \mu \mathrm{m})$ as the stationary phase and using the AR- or HPLC-grade solvents indicated. The melting points of solids 
purified by such means were recorded directly (ie after they had crystallized from the concentrated chromatographic fractions). Starting materials, reagents, drying agents, and other inorganic salts were generally commercially available and were used as supplied. Tetrahydrofuran (THF), methanol and dichloromethane were dried using a solvent purification system that is based upon a technology originally described by Grubbs et al. ${ }^{17}$ Where necessary, reactions were performed under a nitrogen atmosphere.

\section{Specific Chemical Transformations. Methyl 6-((4S,6S)-rel-4-((tert-Butyldimethyl-} silyl)oxy)-6-((4-nitrophenyl)sulfonamido)cyclohex-1-en-1-yl)benzo[d][1,3]dioxole-5-carb

-oxylate (5). A magnetically stirred solution of compound 3 (1.96 g, $4.0 \mathrm{mmol}, 1.0$ equiv.), compound 4 (1.75 g, $6.0 \mathrm{mmol}, 1.5$ equiv.), $\mathrm{PdCl}_{2}(\mathrm{dppf}) \cdot \mathrm{CH}_{2} \mathrm{Cl}_{2}$ (164 mg, $0.22 \mathrm{mmol}, 0.05$ mole equiv.), potassium acetate (1.25 g, $12.74 \mathrm{mmol}, 3.2$ mole equiv.) and cesium carbonate (1.30 g, $4.0 \mathrm{mmol}, 1.0$ mole equiv.) in THF/water (30 mL of a 9:1 v/v mixture) was degassed in a sonicator for $0.33 \mathrm{~h}$. While being maintained under a nitrogen atmosphere, the ensuing mixture was heated under reflux for $3 \mathrm{~h}$ then cooled, quenched with water $(100 \mathrm{~mL})$ and extracted with ethyl acetate $(3 \times 100 \mathrm{~mL})$. The combined organic layers were washed with brine $(1 \times 150 \mathrm{~mL})$ before being dried $\left(\mathrm{MgSO}_{4}\right)$, filtered and concentrated under reduced pressure. The light-yellow oil thus obtained was subjected to flash chromatography (silica gel, 10:0 $\rightarrow$ 7:3 v/v 40-60 petroleum spirits/ethyl acetate gradient elution) to afford, after concentration of the appropriate fractions $\left(R_{\mathrm{f}}=0.7\right.$ in $7: 3 \mathrm{v} / \mathrm{v}$ hexane/ethyl acetate), compound 5 (1.94 g, 83\%) as a white, crystalline solid, m.p. = 175-177 ${ }^{\circ} \mathrm{C} .{ }^{1} \mathrm{H}$ NMR (400 MHz, $\left.\mathrm{CDCl}_{3}\right) \delta 7.66$ (d, $\left.J=7.9 \mathrm{~Hz}, 2 \mathrm{H}\right), 7.52(\mathrm{t}, J=7.6 \mathrm{~Hz}, 1 \mathrm{H}), 7.45(\mathrm{~m}, 1 \mathrm{H}), 7.09$ (s, 1H), 6.58 (m, 1H), 6.31 (s, 1H), 5.88 (s, 1H), $5.86(\mathrm{~s}, 1 \mathrm{H}), 5.40(\mathrm{~s}, 1 \mathrm{H}), 4.49(\mathrm{~m}, 1 \mathrm{H}), 4.23(\mathrm{~s}, 1 \mathrm{H})$, 4.13 (m, 1H), 3.85 (s, 3H), 2.36 (m, 1H), 2.27 (m, 1H), 2.19 (m, 1H), 0.95 (s, 9H), 0.16 (s, 
3H), 0.15 (s, 3H); ${ }^{13} \mathrm{C}$ NMR (100 MHz, $\left.\mathrm{CDCl}_{3}\right) \delta 166.8,149.7,147.0,146.6,138.8,135.6$, 132.5, 131.9, 129.7, 124.8, 124.6, 122.7, 110.8, 109.7, 101.7, 65.9, 53.1, 52.3, 39.3, 34.7, 26.0, 18.3, -4.7 (two signals obscured or overlapping); IR (KBr) $v_{\max } 3374,2928,2856$, 1712, 1541, 1486, 1437, 1407, 1368, 1253, 1165, 1124, 1038, 853, 836, $780 \mathrm{~cm}^{-1}$; MS (ESI, $+\mathrm{ve}) \mathrm{m} / \mathrm{z} 613\left[(\mathrm{M}+\mathrm{Na})^{+}, 100 \%\right]$; HRMS (ESI, +ve) Found: $(\mathrm{M}+\mathrm{Na})^{+}$613.1652, $\mathrm{C}_{27} \mathrm{H}_{34} \mathrm{~N}_{2} \mathrm{NaO}_{9} \mathrm{SSi}$ requires $(\mathrm{M}+\mathrm{Na})^{+} 613.1652$.

Methyl 6-((4S,6S)-rel-6-((N-(But-2-yn-1-yl)-4-nitrophenyl)sulfonamido)-4-((tert-butyldimethylsilyl)oxy)cyclohex-1-en-1-yl)benzo[d][1,3]dioxole-5-carboxylate (6). A magnetically stirred solution of compound 5 (3.29 g, $5.57 \mathrm{mmol}, 1.0$ equiv.) in DMF (50 mL) maintained under a nitrogen atmosphere at $0{ }^{\circ} \mathrm{C}$ was treated with sodium hydride (267 $\mathrm{mg}$ of a $60 \%$ suspension in oil, $6.68 \mathrm{mmol}, 1.2$ equiv.). After $0.33 \mathrm{~h}$ the reaction mixture was treated with 1-bromobut-2-yne (976 $\mu \mathrm{L}, 11.14 \mathrm{mmol}, 2.0$ equiv.) and the mixture thus obtained was allowed to warm to $22{ }^{\circ} \mathrm{C}$ then heated to $60{ }^{\circ} \mathrm{C}$. It was then stirred at this temperature for $6 \mathrm{~h}$ before being cooled then quenched with ice-water $(40 \mathrm{~mL}$ ) (CAUTION: POSSIBILITY OF HYDROGEN GAS EVOLUTION) and diluted with ethyl acetate (50 $\mathrm{mL})$. The separated aqueous layer was extracted with ethyl acetate $(3 \times 50 \mathrm{~mL})$ and the combined organic layers washed with $\mathrm{LiCl}(2 \times 100 \mathrm{~mL}$ of a $5 \% \mathrm{w} / \mathrm{v}$ aqueous solution) then brine $(1 \times 100 \mathrm{~mL})$ before being dried $\left(\mathrm{MgSO}_{4}\right)$, filtered and concentrated under reduced pressure. The resulting light-yellow oil was subjected to flash chromatography (silica gel, 10:0 $\rightarrow$ 7:3 v/v 40-60 petroleum spirits/ethyl acetate gradient elution) to afford, after concentration of the appropriate fractions ( $R_{\mathrm{f}}=0.7$ in $7: 3 \mathrm{v} / \mathrm{v}$ hexane/ethyl acetate), compound 6 (3.33 g, 93\%) as a light-yellow solid, m.p. $=189-191{ }^{\circ} \mathrm{C} .{ }^{1} \mathrm{H}$ NMR $(400 \mathrm{MHz}$, $\left.\mathrm{CDCl}_{3}\right) \delta 8.02(\mathrm{~d}, J=7.9 \mathrm{~Hz}, 1 \mathrm{H}), 7.61(\mathrm{~m}, 1 \mathrm{H}), 7.52(\mathrm{~m}, 1 \mathrm{H}), 7.49$ (dd, $J=7.9$ and $1.4 \mathrm{~Hz}$, 
1H), 7.22 (s, 1H), 6.61 (s, 1H), 5.96 (s, 2H), 5.66 (m, 1H), 5.30 (m, 1H), 4.14-4.01 (complex m, 2H), 3.86 (broad s, 1H), 3.80 (s, 3H), 2.42 (m, 1H), 2.24-2.06 (complex m, 3H), 1.61 (s, 3H), 0.86 (s, 9H), 0.07 (s, 3H), 0.04 (s, 3H); ${ }^{13} \mathrm{C}$ NMR (100 MHz, $\left.\mathrm{CDCl}_{3}\right) \delta$ 166.6, 150.2, 148.1, 146.7, 139.0, 137.4, 133.9, 133.3, 131.7, 130.8, 129.5, 123.5, 123.0, 110.8, 110.2, 101.9, 81.2, 75.1, 67.0, 58.3, 52.0, 37.8, 35.8, 34.6, 25.9, 18.1, 3.5, -4.5, -4.5; IR (KBr) $v_{\max }$ 2953, 2929, 2895, 2856, 1720, 1545, 1485, 1436, 1371, 1249, 1169, 1123, 1102, 1035, 861, 836, $777 \mathrm{~cm}^{-1}$; MS (ESI, +ve) m/z $665\left[(\mathrm{M}+\mathrm{Na})^{+}, 100 \%\right]$; HRMS (ESI, +ve) Found: $(\mathrm{M}+\mathrm{Na})^{+}$665.1964, $\mathrm{C}_{31} \mathrm{H}_{38} \mathrm{~N}_{2} \mathrm{NaO}_{9} \mathrm{SSi}$ requires $(\mathrm{M}+\mathrm{Na})^{+} 665.1965$.

Methyl 6-((rel-3aR,6R,7aS,Z)-rel-6-((tert-Butyldimethylsilyl)oxy)-3-ethylidene-1-((4nitro- phenyl)sulfonyl)-1,2,3,6,7,7a-hexahydro-3aH-indol-3a-yl)benzo[d][1,3]dioxole-5carbox -ylate (7). A magnetically stirred solution of compound 6 (500 mg, $0.78 \mathrm{mmol}, 1.0$ equiv.) in toluene (10 mL) containing $\mathrm{Pd}(\mathrm{OAc})_{2}(35 \mathrm{mg}, 0.16 \mathrm{mmol}, 0.2$ equiv.) and BBEDA (38 mg, $0.16 \mathrm{mmol}, 0.2$ equiv.) was degased in a sonicator for $0.5 \mathrm{~h}$ then heated under reflux overnight. The cooled reaction mixture was concentrated under reduced pressure and the residue thus obtained subjected to flash chromatography (silica gel, 10:0 $\rightarrow$ 7:3 v/v 40-60 petroleum spirits/ethyl acetate gradient elution) to afford, after concentration of the appropriate fractions ( $R_{\mathrm{f}}=0.7 \mathrm{in} 7: 3 \mathrm{v} / \mathrm{v}$ hexane/ethyl acetate), compound 7 (370 mg, 73\%) as a light-yellow solid, m.p. $=175-177{ }^{\circ} \mathrm{C} .{ }^{1} \mathrm{H}$ NMR $\left(400 \mathrm{MHz}, \mathrm{CDCl}_{3}\right) \delta 7.68(\mathrm{~d}, J=8.1$ Hz, 1H), 7.43 (t, $J=7.7 \mathrm{~Hz}, 1 \mathrm{H}), 7.36$ (d, $J=8.1 \mathrm{~Hz}, 1 \mathrm{H}), 7.21$ (t, $J=7.7 \mathrm{~Hz}, 1 \mathrm{H}), 6.63$ (s, 1H), 6.45 (s, 1H), 5.85 (s, 1H), 5.80 (s, 1H), 5.65 (d, $J=10.2 \mathrm{~Hz}, 1 \mathrm{H}), 5.55$ (d, $J=10.2 \mathrm{~Hz}$, 1H), 5.37 (m, 1H), 5.13 (m, 1H), 4.65 (d, $J=15.6 \mathrm{~Hz}, 1 \mathrm{H}), 4.58$ (complex m, 1H), 4.34 (d, $J$ = $15.6 \mathrm{~Hz}, 1 \mathrm{H}), 3.73(\mathrm{~s}, 3 \mathrm{H}), 2.31(\mathrm{~m}, 1 \mathrm{H}), 1.74(\mathrm{~m}, 3 \mathrm{H}), 1.54(\mathrm{~s}, 1 \mathrm{H}), 0.92(\mathrm{~s}, 9 \mathrm{H}), 0.14(\mathrm{~s}$, 3H), 0.13 (s, 3H); ${ }^{13} \mathrm{C}$ NMR (100 MHz, $\left.\mathrm{CDCl}_{3}\right) \delta$ 169.7, 147.9, 147.4, 145.9, 141.9, 137.9, 
132.9, 132.4, 132.1, 131.3, 130.0, 128.9, 126.7, 123.1, 122.9, 109.7, 109.1, 101.7, 67.3, 65.8, 54.3, 52.6, 48.8, 38.5, 26.1, 18.4, 15.0, -4.4, -4.6; IR (KBr) v $v_{\max } 2952,2929,2857,1722$, 1543, 1487, 1435, 1371, 1356, 1247, 1164, 1119, 1071, 1040, 909, 852, 835, 776, $728 \mathrm{~cm}^{-1}$; MS (ESI, +ve) m/z 665 [(M+Na) $\left.{ }^{+}, 100 \%\right]$; HRMS (ESI, +ve) Found: $(\mathrm{M}+\mathrm{Na})^{+} 665.1967$, $\mathrm{C}_{31} \mathrm{H}_{38} \mathrm{~N}_{2} \mathrm{NaO}_{9} \mathrm{SS}$ i requires $(\mathrm{M}+\mathrm{Na})^{+} 665.1965$.

(3R,4aS,5S,11bR,Z)-rel-3-((tert-Butyldimethylsilyl)oxy)-12-ethylidene-4,4a-dihydro-3H, -6H-5,11b-ethano[1,3]dioxolo[4,5-j]phenanthridin-6-one (8). A magnetically stirred solution of compound 7 (224 mg, 0.35 mmol, 1.0 equiv.) in dry DMF (10 mL) containing cesium carbonate (516 mg, $1.58 \mathrm{mmol}, 4.5$ equiv.) and p-chlorothiophenol (211 mg, 1.46 mmol, 4.2 equiv.) was stirred at $22{ }^{\circ} \mathrm{C}$ for $1 \mathrm{~h}$ then quenched with $\mathrm{NH}_{4} \mathrm{Cl}(10 \mathrm{~mL}$ of a saturated aqueous solution) and extracted with dichloromethane $(3 \times 20 \mathrm{~mL})$. The combined organic layers were washed with brine $(1 \times 50 \mathrm{~mL})$ before being dried $\left(\mathrm{MgSO}_{4}\right)$, filtered and concentrated under reduced pressure. The oily residue thus obtained was subjected to flash chromatography (silica gel, 10:0 $\rightarrow$ 8:2 v/v 40-60 petroleum spirits/ethyl acetate gradient elution) to afford, after concentration of the appropriate fractions $\left(R_{\mathrm{f}}=0.7\right.$ in $8: 2 \mathrm{v} / \mathrm{v}$ hexane/ethyl acetate), a white powder. Recrystallization (methanol/dichloromethane/hexane) of this material gave compound 8 (118 mg, 79\%) as a white, crystalline solid, m.p. = 165-167 ${ }^{\circ} \mathrm{C} .{ }^{1} \mathrm{H}$ NMR (400 MHz, $\left.\mathrm{CDCl}_{3}\right) \delta 7.44(\mathrm{~s}, 1 \mathrm{H}), 6.81(\mathrm{~s}, 1 \mathrm{H}), 6.32(\mathrm{dd}, J=10.2$ and $2.3 \mathrm{~Hz}$, 1H), 6.00 (dd, $J=6.2$ and $1.1 \mathrm{~Hz}, 2 \mathrm{H}), 5.80$ (d, $J=10.2,1 \mathrm{H}), 5.33(\mathrm{~m}, 1 \mathrm{H}), 4.37$ (m, 1H), 4.06 (d, $J=16.5 \mathrm{~Hz}, 1 \mathrm{H}), 3.54$ (m, 2H), 1.97 (m, 1H), 1.58 (m, 1H), 1.51 (d, $J=6.9 \mathrm{~Hz}, 3 \mathrm{H})$, 0.89 (s, 9H), 0.08 (s, 3H), 0.07 (s, 3H); ${ }^{13} \mathrm{C}$ NMR (100 MHz, $\left.\mathrm{CDCl}_{3}\right) \delta$ 181.5, 152.8, 147.9, 146.7, 144.1, 134.7, 124.5, 121.6, 119.5, 110.1, 102.4, 102.1, 68.4, 68.0, 51.8, 49.2, 33.9, 26.0, 18.4, 15.3, -4.4, -4.7; IR (KBr) $v_{\max } 2953,2921,2851,1720,1615,1503,1485,1422$, 
1366, 1249, 1118, 1095, 1038, 932, 888, 873, $779 \mathrm{~cm}^{-1}$; MS (ESI, +ve) m/z $426\left[(\mathrm{M}+\mathrm{H})^{+}\right.$, 100\%]; HRMS (ESI, +ve) Found: $(\mathrm{M}+\mathrm{H})^{+}$426.2099, $\mathrm{C}_{24} \mathrm{H}_{32} \mathrm{NO}_{4} \mathrm{Si}$ requires $(\mathrm{M}+\mathrm{H})^{+}$ 426.2101.

(3R,4aS,5S,11bS)-rel-3-((tert-Butyldimethylsilyl)oxy)-4,4a-dihydro-3H,6H-5,11b-ethano -[1,3]dioxolo[4,5-j]phenanthridine-6,12-dione (9). Step i: A magnetically stirred solution of compound 8 (800 mg, $1.86 \mathrm{mmol}, 1.0$ equiv.) in acetonitrile/water (100 mL of a 4:1 v/v mixture) maintained at $22{ }^{\circ} \mathrm{C}$ was treated with citric acid (393 mg, $2.05 \mathrm{mmol}, 1.1$ equiv.), $N$ methylmorpholine $N$-oxide (656 mg, $3.84 \mathrm{mmol}, 2.1$ mole equiv.) and potassium osmate dihydrate (70 mg, $0.19 \mathrm{mmol}, 0.1$ mole equiv.). The ensuing mixture was stirred vigorously at $22{ }^{\circ} \mathrm{C}$ for $3 \mathrm{~h}$ before being diluted with ethyl acetate $(50 \mathrm{~mL})$ and water $(50 \mathrm{~mL})$. The separated aqueous phase was extracted with ethyl acetate $(2 \times 100 \mathrm{~mL})$ and the combined organic phases then washed with brine $(1 \mathrm{x} 100 \mathrm{~mL})$ before being dried $\left(\mathrm{MgSO}_{4}\right)$, filtered and concentrated under reduced pressure. The resulting light-brown oil was used immediately in the next step as detailed immediately below.

Step ii: A solution of the oil obtained as described above (step i) in dichloromethane (10 mL) was treated with iodosobenzene diacetate (663 mg, $2.05 \mathrm{mmol}$, 1.1 equiv.). The ensuing mixture was stirred vigorously at $22{ }^{\circ} \mathrm{C}$ for $2 \mathrm{~h}$ before being treated with TLC-grade silica gel (500 mg) then concentrated under reduced pressure. The resulting free-flowing solid was subjected to flash chromatography (silica gel, 10:0 $\rightarrow$ 8:2 v/v 40-60 petroleum spirits/ethyl acetate gradient elution) and concentration of the appropriate fractions $\left(R_{\mathrm{f}}=0.7\right.$ in $7: 3 \mathrm{v} / \mathrm{v}$ hexane/ethyl acetate) afforded the title compound 9 (392 mg, 51\%) as an unstable, white solid, m.p. $=105-10{ }^{\circ} \mathrm{C}$ (decomposition). ${ }^{1} \mathrm{H}$ NMR $\left(400 \mathrm{MHz}, \mathrm{CDCl}_{3}\right) \delta 7.50(\mathrm{~s}, 1 \mathrm{H}), 6.82$ (s, 1H), 6.22 (d, $J=10.2 \mathrm{~Hz}, 1 \mathrm{H}), 6.06$ (m, 2H), 6.00 (d, $J=10.2 \mathrm{~Hz}, 1 \mathrm{H}), 4.38$ (m, 1H), 3.90 
(broad d, $J=13.7 \mathrm{~Hz}, 1 \mathrm{H}), 3.81$ (d, $J=18.5 \mathrm{~Hz}, 1 \mathrm{H}$ ), 3.30 (d, $J=18.5 \mathrm{~Hz}, 1 \mathrm{H}), 2.21$ (m, 1H), 1.64 (q, $J=12.2 \mathrm{~Hz}, 1 \mathrm{H}), 0.90$ (s, 9H), 0.09 (s, 3H), 0.08 (s, 3H); ${ }^{13} \mathrm{C}$ NMR (100 MHz, $\left.\mathrm{CDCl}_{3}\right) \delta 208.8,179.8,153.8,148.1,139.6,138.4,121.4,120.3,110.2,104.1,102.6,67.7$, 67.2, 53.9, 52.0, 34.4, 25.9, 18.3, -4.5, -4.7; IR (KBr) vmax 2954, 2929, 2858, 1751, 1700, 1485, 1281, 1103, 1089, 1078, 1033, 934, 871, 851, 838, $782 \mathrm{~cm}^{-1}$; MS (EI, +ve) m/z 413 (M+•, 15\%), 356 (18), 329 (30), 328 (100), 298 (60), 253 (55), 225 (99); HRMS (EI, +ve) Found: $\mathrm{M}^{+\bullet} 413.1660, \mathrm{C}_{22} \mathrm{H}_{27} \mathrm{NO}_{5} \mathrm{Si}$ requires $\mathrm{M}^{+\bullet} 413.1659$.

(3R,4aS,6aS,13bS)-rel-3-((tert-Butyldimethylsilyl)oxy)-3,4,4a,5,6,6a-hexahydro-8H-[1,3] -dioxolo[4',5':6,7]isochromeno[3,4-c]indol-8-one (12) and (3R,4aS,5S,11bS,12R)-rel-3((tert-Butyldimethylsilyl)oxy)-12-hydroxy-4,4a-dihydro-3H,6H-11b,5-ethano[1,3]dioxolo [4,5-j]phenanthridin-6-one (13). Step i: A magnetically stirred solution of compound 9 (240 mg, $0.58 \mathrm{mmol}, 1.0$ equiv.) in THF was cooled to $0{ }^{\circ} \mathrm{C}$ then treated with chilled methanol (10 $\mathrm{mL}$ ) followed by sodium borohydride (219 mg, $5.8 \mathrm{mmol}, 10.0$ mole equiv.). The ensuing mixture was warmed to $22^{\circ} \mathrm{C}$, stirred at this temperature for $0.25 \mathrm{~h}$, quenched with $\mathrm{NH}_{4} \mathrm{Cl}(5$ $\mathrm{mL}$ of a saturated aqueous solution) then diluted with ethyl acetate (30 mL) and water (30 $\mathrm{mL}$ ). The separated aqueous phase was extracted with ethyl acetate (2 x $30 \mathrm{~mL}$ ) and the combined organic phases then washed with brine $(1 \times 100 \mathrm{~mL})$ before being dried $\left(\mathrm{MgSO}_{4}\right)$, filtered and concentrated under reduced pressure. The ensuing light-brown oil was subjected to flash chromatography (silica gel, 10:0 $\rightarrow$ 95:5 v/v dichloromethane/ammonia-saturated methanol gradient elution elution) and concentration of the appropriate fractions $\left(R_{\mathrm{f}}=0.5\right.$ in 95:5 v/v dichloromethane/ammonia-saturated methanol) afforded a ca. 3:2 mixture of compounds 10 and 11 (181 mg, 74\% combined yield) as a clear, colorless oil. 
Step ii: A vigorously stirred solution of a ca. 3:2 mixture of compounds 10 and 11 (181 mg, $0.43 \mathrm{mmol}$ ) in dichloromethane $(20 \mathrm{~mL}$ ) was treated with manganese(IV) oxide (377 mg) and the ensuing mixture maintained at $22{ }^{\circ} \mathrm{C}$ for $16 \mathrm{~h}$ then filtered through a pad of diatomaceous earth. The solids thus retained were washed with dichloromethane $(40 \mathrm{~mL})$ and the combined organic filtrates concentrated under reduced pressure. The resulting lightbrown oil was subjected to flash chromatography (silica gel, 10:0 $\rightarrow$ 95:5 v/v dichloromethane/ammonia-saturated methanol gradient elution) to afford two fractions, A and $\mathrm{B}$.

Concentration of fraction A $\left(R_{\mathrm{f}}=0.6\right.$ in $95: 5 \mathrm{v} / \mathrm{v}$ dichloromethane/ammonia-saturated methanol) afforded compound 12 (108 mg, 45\%) as a clear, colorless but rather unstable oil. ${ }^{1} \mathrm{H}$ NMR (400 MHz, $\left.\mathrm{CDCl}_{3}\right) \delta 7.59(\mathrm{~s}, 1 \mathrm{H}), 6.59(\mathrm{~s}, 1 \mathrm{H}), 6.21(\mathrm{~m}, 1 \mathrm{H}), 6.04(\mathrm{ABq}, J=6.2$ Hz, 2H), 5.43 (d, $J=9.9$ Hz, 1H), 4.85 (m, 1H), 4.35 (m, 1H), 3.46 (s, 1H), 3.41 (m, 1H), 3.32 (m, 1H), 2.15 (d, J = 15.3 Hz, 1H), 1.84 (dm, J = $15.3 \mathrm{~Hz}, 1 \mathrm{H}), 0.90$ (s, 9H), 0.13 (s, 3H), 0.12 (s, 3H) (signal due to N-H group proton not observed); ${ }^{13} \mathrm{C}$ NMR (100 MHz, $\left.\mathrm{CDCl}_{3}\right) \delta 163.2,152.7,147.7,136.7,133.4,127.2,117.4,110.2,107.3,102.2,87.4,62.8$, 61.5, 52.2, 48.3, 28.7, 25.9, 18.1, -4.6, -4.7; IR (KBr) $v_{\max } 3366,2928,1710,1617,1481$, 1385, 1273, 1056, 1038, 1017, 836, $778 \mathrm{~cm}^{-1}$; MS (ESI, +ve) m/z $416\left[(\mathrm{M}+\mathrm{H})^{+}, 100 \%\right]$; HRMS (ESI, +ve) Found: $(\mathrm{M}+\mathrm{H})^{+}$416.1890, $\mathrm{C}_{22} \mathrm{H}_{30} \mathrm{NO}_{5} \mathrm{Si}$ requires $(\mathrm{M}+\mathrm{H})^{+} 416.1893$.

Concentration of the fraction B $\left(R_{\mathrm{f}}=0.5\right.$ in $95: 5 \mathrm{v} / \mathrm{v}$ dichloromethane/-saturated methanol $)$ afforded a white powder, recrystallization (dichloromethane/hexane) of which gave compound 13 (71 mg, 29\%) as a white, crystalline solid, m.p. $=191-194{ }^{\circ} \mathrm{C} .{ }^{1} \mathrm{H}$ NMR (400 $\left.\mathrm{MHz}, \mathrm{CDCl}_{3}\right) \delta 7.43(\mathrm{~s}, 1 \mathrm{H}), 6.83(\mathrm{~s}, 1 \mathrm{H}), 6.15(\mathrm{ABq}, J=10.4 \mathrm{~Hz}, 2 \mathrm{H}), 6.02(\mathrm{~s}, 2 \mathrm{H}), 4.39$ (m, 1H), 4.03 (m, 1H), 3.57 (m, 2H), 3.49 (m, 1H), 2.33-1.87 (complex m, 3H), 0.89 (s, 9H), 
0.08 (s, 3H), 0.07 (s, 3H); ${ }^{13} \mathrm{C}$ NMR (100 MHz, $\left.\mathrm{CDCl}_{3}\right) \delta 181.1,152.8,147.0,146.4,140.9$, 121.9, 121.0, 110.2, 103.2, 102.2, 68.0, 67.7, 58.2, 52.1, 33.2, 26.0, 18.4, -4.4, -4.7; IR (KBr) $v_{\max } 3467,2929,2857,1683,1613,1480,1264,1254,1050,1029,803,772,728$ $\mathrm{cm}^{-1}$; MS (ESI, +ve) m/z 416 [(M+H) $\left.{ }^{+}, 100 \%\right]$; HRMS (ESI, +ve) Found: $(\mathrm{M}+\mathrm{H})^{+}$416.1893, $\mathrm{C}_{22} \mathrm{H}_{30} \mathrm{NO}_{5} \mathrm{Si}$ requires $(\mathrm{M}+\mathrm{H})^{+} 416.1893$.

(3R,4aS,6aS,13bS)-rel-3-Hydroxy-3,4,4a,5,6,6a-hexahydro-8H-[1,3]dioxolo[4',5':6,7]isochromeno[3,4-c]indol-8-one (14). A magnetically stirred solution of compound 12 (20 mg, 0.048 mmol, 1.0 mole equiv. $)$ in THF $(1 \mathrm{~mL})$ maintained at $0{ }^{\circ} \mathrm{C}$ was treated with HF•pyridine ( $2 \mu \mathrm{L}, 0.073 \mathrm{mmol}, 1.5$ equiv.) and the ensuing mixture stirred at this temperature for $0.5 \mathrm{~h}$ before being concentrated under reduced pressure. The residue thus obtained was subjected to flash column chromatography (silica gel, 10:0 $\rightarrow$ 95:5 v/v dichloromethane/ammonia-saturated methanol gradient elution) and concentration of the appropriate fractions $\left(R_{\mathrm{f}}=0.4\right.$ in $95: 5 \mathrm{v} / \mathrm{v}$ dichloromethane/ammonia-saturated methanol) gave compound 14 (14 mg, 99\%) as a clear, colorless oil. ${ }^{1} \mathrm{H}$ NMR (400 $\left.\mathrm{MHz}, \mathrm{CDCl}_{3}\right) \delta 7.55$ (s, 1H), 6.64 (s, 1H), 6.49 (m, 1H), 6.04 (m, 2H), 5.46 (d, J = 10.0 Hz, 1H), 4.78 (s, 1H), 4.18 (m, 1H), 3.79 (s, 1H), 3.33 (m, 2H), $2.16(\mathrm{~m}, 1 \mathrm{H}), 1.75(\mathrm{dm}, J=14.8 \mathrm{~Hz}, 1 \mathrm{H})$ (signals due to $\mathrm{N}-\mathrm{H}$ and $\mathrm{O}-\mathrm{H}$ group protons not observed); ${ }^{13} \mathrm{C} \mathrm{NMR}\left(100 \mathrm{MHz}, \mathrm{CDCl}_{3}\right) \delta 163.3,152.9$, 147.9, 136.9, 136.0, 125.5, 116.8, 110.2, 107.6, 102.3, 87.4, 63.2, 60.5, 51.0, 48.2, 29.1; IR (KBr) $v_{\max } 3329,2919,1705,1616,1480,1440,1383,1272,1243,1115,1057,1035,931$, 909, $728 \mathrm{~cm}^{-1}$; MS (ESI, +ve) m/z 324 (20\%), 302 [(M+H) $\left.{ }^{+}, 100\right]$; HRMS (ESI, +ve) Found: $(\mathrm{M}+\mathrm{H})^{+}$302.1026, $\mathrm{C}_{16} \mathrm{H}_{16} \mathrm{NO}_{5}$ requires $(\mathrm{M}+\mathrm{H})^{+} 302.1028$.

(3R,4aS,6aS,13bS)-rel-3-Hydroxy-5-methyl-3,4,4a,5,6,6a-hexahydro-8H-[1,3]dioxolo[4',5':6,7]isochromeno[3,4-c]indol-8-one [( \pm )-3-O-Demethyl-6-epi-macronine, 15]. A mag 
-netically stirred solution of compound 14 (10 mg, $0.033 \mathrm{mmol})$ in THF (1 mL) maintained under a nitrogen atmosphere at $0{ }^{\circ} \mathrm{C}$ was treated with potassium hydride (17.6 mg of a $30 \mathrm{wt}$ \% dispersion in mineral oil, $0.13 \mathrm{mmol}, 4.0$ mole equiv.) then methyl iodide (16.5 $\mu \mathrm{L}, 0.27$ mmol, 4.0 mole equiv.). The ensuing mixture was stirred at $0{ }^{\circ} \mathrm{C}$ for $0.5 \mathrm{~h}$ before being quenched with water $(5 \mathrm{~mL})$ (CAUTION: POSSIBILITY OF EVOLUTION OF HYDROGEN) then extracted with ethyl acetate $(3 \times 10 \mathrm{~mL})$. The combined organic phases were washed with brine $(1 \times 50 \mathrm{~mL})$ then dried $\left(\mathrm{Na}_{2} \mathrm{SO}_{4}\right)$, filtered, and concentrated under reduced pressure. The residue thus obtained was subjected to flash column chromatography (silica gel, 10:0 $\rightarrow$ 95:5 v/v dichloromethane/ammonia-saturated methanol gradient elution) and concentration of the appropriate fractions $\left(R_{\mathrm{f}}=0.5\right.$ in $95: 5 \mathrm{v} / \mathrm{v}$ dichloromethane/ammonia-saturated methanol) afforded compound 15 (10 mg, 99\%) as a clear, colorless oil. ${ }^{1} \mathrm{H}$ NMR (400 MHz, $\left.\mathrm{CDCl}_{3}\right) \delta 7.58(\mathrm{~s}, 1 \mathrm{H}), 6.65$ (s, 1H), 6.46 (m, 1H), 6.05 (ABq, $J=4.3 \mathrm{~Hz}, 2 \mathrm{H}), 5.45$ (d, $J=9.9 \mathrm{~Hz}, 1 \mathrm{H}), 4.73$ (s, 1H), 4.15 (broad s, 1H), 3.59 (dd, $J=13.0$ and $3.2 \mathrm{~Hz}, 1 \mathrm{H}), 3.14$ (s, 1H), 2.95 (d, $J=13.0 \mathrm{~Hz}, 1 \mathrm{H}$ ), 2.58 (s, 3H), 2.20 (broad d, $J=14.9 \mathrm{~Hz}, 1 \mathrm{H}$ ), 1.73 (broad d, $J=14.9 \mathrm{~Hz}, 1 \mathrm{H}$ ) (signal due to O-H group proton not observed); ${ }^{13} \mathrm{C} \mathrm{NMR}\left(100 \mathrm{MHz}, \mathrm{CDCl}_{3}\right) \delta 163.1,152.9,147.9,136.6,135.4,125.6$, 117.1, 110.2, 107.7, 102.4, 86.4, 68.9, 63.3, 59.9, 49.1, 42.7, 26.8; IR (KBr) vmax 3420, 2919, 1716, 1617, 1482, 1440, 1275, 1247, 1127, 1055, 1036, $932 \mathrm{~cm}^{-1}$; MS (ESI, +ve) m/z 338 (69\%), $316\left[(\mathrm{M}+\mathrm{H})^{+}, 100\right]$; HRMS (ESI, +ve) Found: $(\mathrm{M}+\mathrm{H})^{+} 316.1183, \mathrm{C}_{17} \mathrm{H}_{18} \mathrm{NO}_{5}$ requires $(\mathrm{M}+\mathrm{H})^{+} 316.1185$.

(3R,4aS,6aR,13bS)-rel-3-Hydroxy-3,4,4a,5,6,6a-hexahydro-8H-[1,3]dioxolo[4',5' :6,7]isochromeno[3,4-c]indol-8-one (16). A magnetically stirred solution of compound 13 (120 mg, $0.29 \mathrm{mmol})$ in toluene $(5.0 \mathrm{~mL})$ was treated with $p$-toluenesulfonic acid monohydrate $(54.9$ 
$\mathrm{mg}, 0.33 \mathrm{mmol}$, 1.1 equiv.) and the resulting solution stirred at $90{ }^{\circ} \mathrm{C}$ for $1.5 \mathrm{~h}$ then cooled, quenched with $\mathrm{NaHCO}_{3}(10 \mathrm{~mL}$ of a saturated aqueous solution) and extracted with ethyl acetate $(3 \times 20 \mathrm{~mL})$. The combined organic phases were washed with brine $(1 \times 100 \mathrm{~mL})$ then dried $\left(\mathrm{Na}_{2} \mathrm{SO}_{4}\right)$, filtered, and concentrated under reduced pressure. The residue thus obtained was subject to flash column chromatography (silica gel, 10:0 $\rightarrow$ 95:5 v/v dichloromethane/ammonia-saturated methanol gradient elution) and concentration of the appropriate fractions $\left(R_{\mathrm{f}}=0.4\right.$ in 95:5 v/v dichloromethane/ammonia-saturated methanol) afforded compound 16 (42 mg, 48\%) as a white, crystalline solid, m.p. $=181-184{ }^{\circ} \mathrm{C} .{ }^{1} \mathrm{H}$ NMR (400 MHz, $\left.\mathrm{CDCl}_{3}\right) \delta 7.55$ (s, 1H), 6.66 (s, 1H), 6.23 (m, 1H), 6.05 (ABq, J = $8.0 \mathrm{~Hz}$, 2H), 5.63 (d, $J=10.2 \mathrm{~Hz}, 1 \mathrm{H}), 4.56(\mathrm{~m}, 1 \mathrm{H}), 4.20$ (m, 1H), 4.00 (broad s, 1H), 3.28 (m, 1H), $3.08(\mathrm{t}, J=10.8 \mathrm{~Hz}, 1 \mathrm{H}), 2.46(\mathrm{~d}, J=15.1 \mathrm{~Hz}, 1 \mathrm{H}), 2.15$ (dd, $J=15.1$ and $4.8 \mathrm{~Hz}, 1 \mathrm{H})$ (signals due to $\mathrm{O}-\mathrm{H}$ and $\mathrm{N}-\mathrm{H}$ group protons not observed); ${ }^{13} \mathrm{C}$ NMR (100 $\left.\mathrm{MHz}, \mathrm{CDCl}_{3}\right) \delta$ 165.1, 152.7, 147.7, 140.9, 132.9, 125.7, 118.4, 111.4, 103.7, 102.4, 82.9, 62.7, 55.6, 44.5, 44.0, 32.8; IR (KBr) $v_{\max } 3335,2921,1719,1615,1479,1271,1246,1072,1033,907,727$ $\mathrm{cm}^{-1}$; MS (ESI, +ve) m/z $302\left[(\mathrm{M}+\mathrm{H})^{+}, 100 \%\right]$; HRMS (ESI, +ve) Found: $(\mathrm{M}+\mathrm{H})^{+}$302.1026, $\mathrm{C}_{16} \mathrm{H}_{16} \mathrm{NO}_{5}$ requires $(\mathrm{M}+\mathrm{H})^{+} 302.1028$.

(3R,4aS,6aR,13bS)-rel-3-Hydroxy-5-methyl-3,4,4a,5,6,6a-hexahydro-8H-[1,3]dioxolo$\left[4^{\prime}, 5^{\prime}: 6,7\right]$ isochromeno[3,4-c]indol-8-one $\quad[( \pm)$-3-O-Demethylmacronine, $\quad 2] . \quad \mathrm{A}$ magnetically stirred solution of compound $16(7 \mathrm{mg}, 0.023 \mathrm{mmol})$ in acetonitrile $(1 \mathrm{~mL})$ maintained at $22{ }^{\circ} \mathrm{C}$ was treated, successively, with formaldehyde $(17.5 \mu \mathrm{L}$ of a $37 \%$ aqueous solution, $0.21 \mathrm{mmol}, 9$ mole equiv.), acetic acid (5 $\mu \mathrm{L}, 0.09 \mathrm{mmol}, 3.8$ mole equiv.) and $\mathrm{NaBH}_{3} \mathrm{CN}$ (5.7 mg, $0.87 \mathrm{mmol}, 38$ mole equiv.). The ensuing mixture was stirred at ambient temperatures for $1 \mathrm{~h}$ then quenched with $\mathrm{NaHCO}_{3}$ ( $5 \mathrm{~mL}$ of a saturated aqueous solution) 
before being extracted with ethyl acetate $(3 \times 15 \mathrm{~mL})$. The combined organic phases were washed with brine $(1 \times 50 \mathrm{~mL})$ then dried $\left(\mathrm{Na}_{2} \mathrm{SO}_{4}\right)$ and filtered before being concentrated under reduced pressure. The residue thus obtained was subjected to flash column chromatography (silica gel, 10:0 $\rightarrow$ 95:5 v/v dichloromethane/ammonia-saturated methanol gradient elution) and concentration of the appropriate fractions $\left(R_{\mathrm{f}}=0.5\right.$ in $95: 5 \mathrm{v} / \mathrm{v}$ dichloromethane/ammonia-saturated methanol) afforded a powder that was recrystallized (methanol/water) to give compound $( \pm)-2(6 \mathrm{mg}, 89 \%)$ as a white, crystalline solid, m.p. $=$ 199-202 ${ }^{\circ} \mathrm{C} .{ }^{1} \mathrm{H}$ NMR $\left(400 \mathrm{MHz}, \mathrm{CD}_{3} \mathrm{OD}\right) \delta 7.43(\mathrm{~s}, 1 \mathrm{H}), 6.84(\mathrm{~s}, 1 \mathrm{H}), 6.14(\mathrm{dd}, J=10.1$ and $5.2 \mathrm{~Hz}, 1 \mathrm{H}), 6.08(\mathrm{ABq}, J=1.0 \mathrm{~Hz}, 2 \mathrm{H}), 5.57(\mathrm{dd}, J=10.2$ and $1.4 \mathrm{~Hz}, 1 \mathrm{H}), 4.71(\mathrm{~m}$, 1H), 4.12 (m, 1H), 3.37 (m, 1H), 3.25 (m, 1H), 2.90 (m, 1H), 2.58 (s, 3H), 2.44 (m, 1H), 2.23 (m, 1H) (signal due to $\mathrm{O}-\mathrm{H}$ group proton not observed); ${ }^{13} \mathrm{C}$ NMR (100 MHz, $\left.\mathrm{CD}_{3} \mathrm{OD}\right) \delta$ 167.2, 154.3, 148.9, 143.1, 133.1, 127.2, 119.4, 111.3, 104.9, 103.9, 82.0, 65.6, 64.4, 53.7, 47.5, 42.9, 31.1; IR (KBr) $v_{\max }$ 3267, 2920, 2877, 2852, 1723, 1615, 1480, 1276, 1247, 1026 $\mathrm{cm}^{-1}$; MS (ESI, +ve) m/z 316 [(M+H) $\left.{ }^{+}, 100 \%\right], 298$ (80); HRMS (ESI, +ve) Found $(\mathrm{M}+\mathrm{H})^{+}$, 316.1182. $\mathrm{C}_{17} \mathrm{H}_{18} \mathrm{NO}_{5}$ requires $(\mathrm{M}+\mathrm{H})^{+}$, 316.1185 .

\section{Crystallographic Studies}

\section{Crystallographic Data for Compound 8}

$\mathrm{C}_{24} \mathrm{H}_{31} \mathrm{NO}_{4} \mathrm{Si}, M=425.60, T=150 \mathrm{~K}$, orthorhombic, space group Fdd2, $\mathrm{Z}=16, a=34.4510$ (4) $\AA, b=27.1978$ (4) $\AA, c=9.5573$ (1) $\AA ; V=8955.11(19) \AA^{3}, D_{x}=1.263 \mathrm{~g} \mathrm{~cm}^{-3}, 3717$ unique data $\left(2 \theta_{\max }=144.6^{\circ}\right), R=0.034$ [for 3632 reflections with $I>2.0 \sigma(I)$ ]; $R w=0.089$ (all data), $S=1.0$. 


\section{Crystallographic Data for Compound 13}

$\mathrm{C}_{22} \mathrm{H}_{29} \mathrm{NO}_{5} \mathrm{Si} \cdot \mathrm{CH}_{2} \mathrm{Cl}_{2}, M=500.48, T=150 \mathrm{~K}$, monoclinic, space group $P 21 / c, Z=4, a=$

6.24090 (5) $\AA, b=50.3641$ (3) $\AA, c=8.25660$ (6) $\AA ; \beta=103.2764$ (7) ${ }^{\circ}, V=2525.83$ (3) $\AA^{3}$,

$D_{x}=1.316 \mathrm{~g} \mathrm{~cm}^{-3}, 5129$ unique data $\left(2 \theta_{\max }=147.8^{\circ}\right), R=0.051$ [for 4820 reflections with $I$

$>2.0 \sigma(I)] ; R w=0.129$ (all data), $S=1.5$.

\section{Structure Determination}

Images were measured on a CCD diffractometer $(\mathrm{CuK} \alpha$, mirror monochromator, $\lambda=$ $1.54184 \AA$ ) and data extracted using the CrysAlis PRO package. ${ }^{18}$ Structure solution was by direct methods (SIR92). ${ }^{19}$ The structures of compounds $\mathbf{8}$ and $\mathbf{1 3}$ were refined using the CRYSTALS program package. ${ }^{20}$ Atomic coordinates, bond lengths and angles, and displacement parameters for compound $\mathbf{8}$ and $\mathbf{1 3}$ have been deposited at the Cambridge Crystallographic Data Centre (CCDC no. 1531843 and 1531844, respectively). These data can be obtained free-of-charge via www.ccdc.cam.ac.uk/data_request/cif by emailing data_request@ccdc.cam.ac.uk, or by contacting The Cambridge Crystallographic Data Centre, 12 Union Road, Cambridge CB2 1EZ, UK; fax: +44 1223336033. 


\section{ASSOCIATED CONTENT}

\section{Supporting Information}

The Supporting Information is available free of charge on the ACS Publications website at DOI: 10.1021/acs.joc.XXXXXXX.

Crystallographic data for $\mathbf{8}$ (CIF)

Crystallographic data for $\mathbf{1 3}$ (CIF)

Crystallographic data and anisotropic displacement ellipsoid plots derived from the singlecrystal X-ray analyses of compounds 8 and 13. Tabular comparison of the ${ }^{13} \mathrm{C}$ NMR data reported for 3-O-demethylmacronine with those recorded on the synthetically derived compound ( \pm )-2. ${ }^{1} \mathrm{H}$ and ${ }^{13} \mathrm{C}$ NMR spectra of compounds 5-9, 12-16 and ( \pm )-2 (PDF).

\section{AUTHOR INFORMATION}

Corresponding Author

*E-mail: Martin.Banwell@anu.edu.au.

Notes

The authors declare no competing financial interest.

\section{ACKNOWLEDGEMENTS}

We thank the Australian Research Council for financial support. X.M. is the grateful recipient of a PhD Scholarship provided by the Guangzhou Elite Project of the Guangzhou Municipal Government, People’ s Republic of China. 


\section{REFERENCES}

1. Hauth, H.; Stauffacher, D. Helv. Chem. Acta, 1964, 47, 185.

2. (a) Murphy, C. F.; Wildman, W. C. Tetrahedron Lett., 1964, 3857; (b) Wildman, W. C.; Bailey, D. T. J. Org. Chem., 1968, 33, 3749.

3. Unver, N.; Noyan, S.; Gözler, T.; Onur, M. A.; Gözler, B.; Hesse, M. Planta Med., 1999, 65, 347.

4. (a) Lin, L.-Z.; Hu, S.-F.; Chai, H.-B.; Pengsuparp, T.; Pezzuto, J. M.; Cordell, G. A.; Ruangrungsi, N. Phytochem., 1995, 40, 1295; (b) Berkov, S.; Bastida, J.; Sidjimova, B.; Viladomat, F.; Codina, C. Biochem. Sys. Ecol., 2008, 36, 638; (c) Berkov, S.; Bastida, J.; Sidjimova, B.; Viladomat, F.; Codina, C. Chem. Biodiv., 2011, 8, 115; (d) Iannello, C.; Bastida, J.; Bonvicini, F.; Antognoni, F.; Gentilomi, G. A.; Poli, F. Nat. Prod. Res., 2014, 28, 704; (e) Cortes, N.; Posada-Duque, R. A.; Alvarez, R.; Alzate, F.; Berkov, S.; Cardona-Gómez, G. P.; Osoria, E. Life Sci., 2015, 122, 42; (f) Guerrieri, C. G.; Pigni, N. B.; de Andrade, J. P.; dos Santos, V. D.; Binns, F.; de Souza Borges, W.; Viladomat, F.; Bastida, J. Arab. J. Chem., 2016, 9, 688.

5. $\quad$ Isobe, K.; Taga, J.; Tsuda, Y. Tetrahedron Lett., 1976, 2331.

6. Gao, N. (Y).; Banwell, M. G.; Willis, A. C. Org. Lett., 2017, 19, 162.

7. $\quad$ Petit, L.; Banwell, M. G.; Willis, A. C. Org. Lett., 2011, 13, 5800.

8. $\quad$ Crisp, A. L.; Li, J.; Lan, P.; Nugent, J.; Matoušová, E.; Banwell, M. G. Aust. J. Chem., 2015, 68, 1183.

9. $\quad$ Fukuyama, T.; Jow, C.-K.; Cheung, M. Tetrahedron Lett., 1995, 36, 6373.

10. Ell, A.; Closson, A.; Adolfsson, H.; Bäckvall, J.-E. Adv. Synth. Catal., 2003, 345, 1012. 
11. Nicolaou, K. C.; Adsool, V. A.; Hale, C. R. H. Org. Lett., 2010, 12, 1552.

12. $\mathrm{K}$

R\$QAdV.J2015, \$, 60106. R. G.; Savanur, H. M.

13. Bürgi, H. B.; Dunitz, J. D.; Lehn, J. M.; Wipff, G. Tetrahedron, 1974, 1563.

14. For a useful review on the chemistry of bridged lactams and related heterocycles see Szostak, M.; Aubé, J. Chem. Rev., 2013, 113, 5701.

15. The following reagent/solvent combinations were used in attempts to effect this $O$ methylation: (i) MeI, NaH, THF; (ii) MeI, NaH, DMF; (iii) MeI, $\mathrm{Ag}_{2} \mathrm{O}, \mathrm{THF}$; (iv) $(\mathrm{MeO})_{2} \mathrm{SO}_{2}, \mathrm{~K}_{2} \mathrm{CO}_{3}$, THF. Either no reaction or decomposition of the substrate was observed in each instance.

16. Still, W. C.; Kahn, M.; Mitra, A. J. Org. Chem. 1978, 43, 2923.

17. Pangborn, A. B.; Giardello, M. A.; Grubbs, R. H.; Rosen, R. K.; Timmers, F. J. Organometallics 1996, 15, 1518.

18. DENZO-SMN. Otwinowski, Z.; Minor, W. Processing of X-ray diffraction data collected in oscillation mode. In Methods in Enzymology, Volume 276: Macromolecular Crystallography, Part A; Carter Jr., C. W.; Sweet, R. M. Eds.; Academic Press: New York, 1997; pp. 307-326.

19. CrysAlis PRO Version 1.171.37.35h (released 09-02-2015 CrysAlis171.NET) (compiled Feb 9 2015,16:26:32) Agilent Technologies: Oxfordshire, UK.

20. SIR92. Altomare, A.; Cascarano, G.; Giacovazzo, C.; Guagliardi, A.; Burla, M. C.; Polidori, G.; Camalli, M. J. Appl. Crystallogr. 1994, 27, 435. 